\title{
The effect of hyperfiltration mechanism on kidney function in Living-Donor Kidney Transplantation in Cipto Mangunkusumo Hospital, Jakarta, Indonesia: study on renal arterial resistive index, urinary vascular endothelial growth factor, neutrophil gelatinase- associated lipocalin, and heparan sulfate
}

\author{
Maruhum Bonar Marbun ${ }^{1}$, Endang Susalit ${ }^{1}$, Diana Aulia $^{2}$, Jacub Pandelaki ${ }^{3}$, Saptawati Bardosono ${ }^{4}$, Bambang Purwanto ${ }^{5}$
}

\author{
${ }^{1}$ Department of Internal Medicine, Cipto Mangunkusumo Hospital, Jakarta, Indonesia \\ ${ }^{2}$ Department of Clinical Pathology, Cipto Mangunkusumo Hospital, Jakarta, Indonesia \\ ${ }^{3}$ Department of Radiology, Cipto Mangunkusumo Hospital, Jakarta, Indonesia \\ ${ }^{4}$ Department of Nutrition, Cipto Mangunkusumo Hospital, Jakarta, Indonesia \\ ${ }^{5}$ Department of Internal Medicine, Faculty of Medicine Universitas Sebelas Maret, Surakarta, Indonesia
}

Background: While adequate donors' kidney function after donation is desired, some may recover incompletely and develop chronic kidney diseases. Hyperfiltration is an adapting mechanism to overcome nephrectomy-related kidney function loss. This study aims to explain adaptive and maladaptive hyperfiltration mechanism during the first 30 days postnephrectomy.

Methods: Longitudinal observational study was conducted on 46 kidney donors in Cipto Mangunkusumo Hospital in April-December 2019. Estimated glomerular filtration rate (eGFR) and albumin-creatinine ratio (ACR) were examined serially. Subjects were divided into adaptive (eGFR $>60 \mathrm{~mL} / \mathrm{min} / 1.73 \mathrm{~m}^{2}$ and/or ACR $<30 \mathrm{mg} / \mathrm{g}$ on day 30) and maladaptive group (eGFR $<60 \mathrm{~mL} /$ $\mathrm{min} / 1.73 \mathrm{~m}^{2}$ and/or $\mathrm{ACR}>30 \mathrm{mg} / \mathrm{g}$ on day 30 ). Kidney resistive index (RI), urinary vascular endothelial growth factor (VEGF), and heparan sulfate (HS) were examined prenephrectomy and on day-2, day-7, and day-30 postnephrectomy. Urinary neutrophil gelatinase-associated lipocalin (NGAL) level was measured before and 6-hour postnephrectomy. Donors' characteristic variables were analyzed using chi-square or Fisher test and logistic regression. Difference of RI, VEGF, NGAL, and HS between two groups were analyzed using Mann-Whitney or independent t-test.

Results: Out of 46 subjects, 40 were included in the final analysis. Nineteen donors (47.5\%) underwent maladaptive hyperfiltration. Prenephrectomy eGFR was significantly different with value of $111.17(11.38) \mathrm{mL} / \mathrm{min} / 1.73 \mathrm{~m}^{2}$ and 92.94 (13.21) $\mathrm{mL} /$ $\mathrm{min} / 1.73 \mathrm{~m}^{2}$ and cutoff of $104.60 \mathrm{~mL} / \mathrm{min} / 1.73 \mathrm{~m}^{2}$. Outcomes of donors aged $>45$ years and arterial stiffness $>50$ th percentile were significantly different $(P=0.03$ and $P=0.01)$. Hyperfiltration was evidenced by significant changes in $R I, V E G F, N G A L$, and HS after nephrectomy. Significant difference between arcuate artery RI on day-2 and day-30 was found only in adaptive group.

Conclusions: Hyperfiltration does not alter kidney function on day-30 postnephrectomy. Prenephrectomy eGFR, age $>45$ years, and arterial stiffness are associated with day-30 postnephrectomy kidney function. RI of arcuate artery changes more prominently and rapidly in adaptive group. Further study evaluating kidney function and interfering variables within longer period is necessary.

Corresponding author: Maruhum Bonar Marbun

E-mail: mbhmarbun@gmail.com

This is an Open Access article distributed under the terms of the Creative Commons Attribution Non-Commercial License (http://creativecommons.org/licenses/by-nc/4.0/) which permits unrestricted non-commercial use, distribution, and reproduction in any medium, provided the original work is properly cited. 